\title{
CLIFFORD DIVISION ALGEBRAS AND ANISOTROPIC QUADRATIC FORMS: TWO COUNTEREXAMPLES
}

\author{
by P. MAMMONE and J. P. TIGNOL
}

(Received 22 August, 1985)

In a recent paper [3], D. W. Lewis proposed the following conjecture. (The notation is the same as that in [2] and [3].)

Conjecture. Let $F$ be a field of characteristic not 2 and let $a_{1}, b_{1}, \ldots, a_{n}, b_{n} \in F^{\times}$. The tensor product of quaternion algebras

$$
\left(\begin{array}{cc}
a_{1} b_{1} \\
F
\end{array}\right) \otimes_{F} \ldots \otimes_{F}\left(\begin{array}{cc}
a_{n} & b_{n} \\
F
\end{array}\right)
$$

is a division algebra if and only if the quadratic form over $F$

$$
\perp_{i=1}^{n}(-1)^{i+1}\left\langle a_{i}, b_{i},-a_{i} b_{i}\right\rangle
$$

is anisotropic.

This equivalence indeed holds for $n=1$ as is well known [2, Theorem 2.7], and Albert [1] (see also [4, \$15.7]) has shown that it also holds for $n=2$. The aim of this note is to provide counterexamples to both of the implications for $n \geqslant 3$.

Let $k$ be a field of characteristic different from 2 , and let $x_{1}, \ldots, x_{n-1}, y_{1}, \ldots, y_{n}$ be independent indeterminates over $k$ (with $n \geqslant 3$ ). Let also $f\left(x_{1}, x_{2}\right) \in k\left(x_{1}, x_{2}\right)$ and

$$
F=k\left(x_{1}, \ldots, x_{n-1}, y_{1}, \ldots, y_{n}\right) \text {. }
$$

THEOREM. (1) The tensor product of quaternion algebras

$$
T=\left(\begin{array}{cc}
x_{1} & y_{1} \\
F
\end{array}\right) \otimes \ldots \otimes\left(\begin{array}{cc}
x_{n-1} & y_{n-1} \\
F
\end{array}\right) \otimes\left(\begin{array}{c}
f\left(x_{1}, x_{2}\right) \\
y_{n}
\end{array}\right)
$$

is a division algebra if and only if $f\left(x_{1}, x_{2}\right)$ is not a square in $k\left(\sqrt{x_{1}}, \sqrt{x_{2}}\right)$.

(2) The quadratic form over $F$

$$
\begin{aligned}
Q=\left\langle x_{1}, y_{1},-\right. & \left.x_{1} y_{1}\right\rangle \perp \ldots \perp(-1)^{n} \\
& \times\left\langle x_{n-1}, y_{n-1},-x_{n-1} y_{n-1}\right\rangle \perp(-1)^{n+1}\left\langle f\left(x_{1}, x_{2}\right), y_{n},-f\left(x_{1}, x_{2}\right) y_{n}\right\rangle
\end{aligned}
$$

is anisotropic if and only if $(-1)^{n} f\left(x_{1}, x_{2}\right)$ is not represented by the quadratic form $\left\langle x_{1},-x_{2}\right\rangle$ over $k\left(x_{1}, x_{2}\right)$ and $f\left(x_{1}, x_{2}\right)$ is not a square in $k\left(x_{1}, x_{2}\right)$.

The proof will follow by repeated use of the following results.

LEMMA. Let $K$ be a field of characteristic different from 2 and let $t$ be an indeterminate over $K$.

(1) If $A$ is a central simple algebra over $K$ and $c \in K^{\times}$, then $A \otimes_{K}\left({ }^{c}{ }_{K(t)}{ }^{\prime}\right)$ is a division algebra if and only if $A \otimes_{K} K(\sqrt{c})$ is a division algebra.

Glasgow Math. J. 28 (1986) 227-228. 
(2) If $q_{1}$ and $q_{2}$ are quadratic forms over $K$, then $q_{1} \perp\langle t\rangle q_{2}$ is anisotropic over $K(t)$ if and only if $q_{1}$ and $q_{2}$ are anisotropic over $K$.

Proof. (1) See [5, Proposition 2.4]; (2) see [2, p. 273].

Proof of the theorem. (1) We apply part (1) of the lemma $(n-1)$ times, taking successively $t=y_{1}, t=y_{2}, \ldots, t=y_{n-1}$. It follows that $T$ is a division algebra if and only if

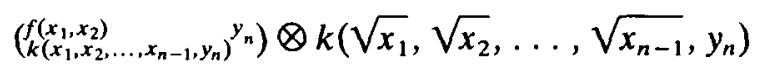

is a division algebra. This last condition is equivalent to the following: the quadratic form

$$
\left\langle 1,-f\left(x_{1}, x_{2}\right)\right\rangle \perp-\left\langle y_{n}\right\rangle\left\langle 1,-f\left(x_{1}, x_{2}\right)\right\rangle
$$

is anisotropic over $k\left(\sqrt{x_{1}}, \sqrt{x_{2}}, \ldots, \sqrt{x_{n-1}}, y_{n}\right)$. Applying then the second part of the lemma with successively $t=y_{n}, \sqrt{x_{n-1}}, \ldots, \sqrt{x_{3}}$, we see that this condition holds if and only if $\left\langle 1,-f\left(x_{1}, x_{2}\right)\right\rangle$ is anisotropic over $k\left(\sqrt{x_{1}}, \sqrt{x_{2}}\right)$, i.e. $f\left(x_{1}, x_{2}\right)$ is not a square in $k\left(\sqrt{x_{1}}, \sqrt{x_{2}}\right)$.

(2) readily follows from the second part of the lemma, applied successively with $t=y_{1}, y_{2}, \ldots, y_{n}, x_{3}, x_{4}, \ldots, x_{n-1}$.

Now, for $f\left(x_{1}, x_{2}\right)=(-1)^{n}\left(x_{1}-x_{2}\right)$, the theorem shows that the tensor product $T$ is a division algebra, while the corresponding quadratic form $Q$ is isotropic.

Conversely, for $f\left(x_{1}, x_{2}\right)=x_{1} x_{2}$, the tensor product $T$ is not a division algebra, but the corresponding quadratic form $Q$ is anisotropic, since part (2) of the lemma, with $t=x_{2}$, shows that $\left\langle x_{1},-x_{2},(-1)^{n} x_{1} x_{2}\right\rangle$ is anisotropic over $k\left(x_{1}, x_{2}\right)$.

\section{REFERENCES}

1. A. A. Albert, A construction of non-cyclic normal division algebras, Bull. Amer. Math. Soc. 38 (1932), 449-456.

2. T.-Y. Lam, The algebraic theory of quadratic forms (Benjamin, 1973).

3. D. W. Lewis, A note on Clifford algebras and central division algebras with involution, Glasgow Math. J. 26 (1985), 171-176.

4. R. S. Pierce, Associative algebras (Springer, 1982).

5. J. P. Tignol, Algèbres indécomposables d'exposant premier, to appear in Adv. in Maths.

Université de Mons.Hainaut

B-7000 MoNS
Université Catholique de Louvain

B-1348 Louvain-LA-NEUVE 